maximum phase of the optical eclipse: this may have been due to asymmetry of noise sources on the solar disk. From 1920 until 1932 G.M.T., no signals were audible on antennæ bearing $082^{\circ}$ directed towards the station $G S D$ except sharp pips of highintensity noise covering a frequency range $9 \cdot 5-$ $11.1 \mathrm{Mc} . / \mathrm{s}$. 'These noise 'pips' reached a maximum in both intensity and frequency of occurrence at 1925 G.M.T., and were characteristic of the noise usually associated with sunspots and normally only heard on much higher frequencies. Reception of this type of noise on frequencies so low as $9.5 \mathrm{Mc} / \mathrm{s}$. therefore indicates a substantial drop in obliqueincidence critical frequency coinciding with the time of maximum phase of the eclipse over that portion of the ionosphere. This does not explain why this noise should have cut off sharply at the upper terminal frequency of $11.1 \mathrm{Mc} / \mathrm{s}$., unless it was radiated on a limited spectrum, as appears to be the case with the large bursts of noise associated with solar flares.

The results obtained from these observations confirm those of previous observers in that they show that ultra-violet radiation from the sun is the main cause of atmospheric ionization, and that when this radiation is intercepted by the moon, the density of ionization and the critical frequencies of the ionized layers decrease. The results of the observations of noise are of interest, though not conclusive, and indicate that this aspect of the effects of an eclipse should receive greater attention when the opportunity again arises.

\section{PAUL EHRLICH}

$\mathrm{O}$ December 5, 1951, the New York Academy of Medicine celebrated the deposition at the Academy of memorabilia of Paul Ehrlich, which were the gift of the Schwerin family. The president, Dr. Wm. Barclay Parsons, receiving the memorabilia on behalf of the Academy, described the Academy's library of 270,000 volumes and numerous pamphlets and its valuable historical treasures. Prof. Perrin $H$. Long, professor of medicine in the State University of New York, summarized Ehrlich's scientific work, and Prof. S. A. Waksman, chairman of the Department of Microbiology, Rutgers University, who graduated in 1915, the year of Ehrlich's death, gave an interesting account of Ehrlich's character and development as a man of science.

A brilliant scholar at school, Ehrlich was extremely modest and escaped when he could to the room at home that he had fixed up as a laboratory for chemical experiments with dyes and for his biological collections. His later work with Koch on tuberculosis, which Ehrlich himself contracted, his work on diphtheria antitoxin with von Behring (full credit for which was not given to Ehrlich at the time), and the development of his well-known later work, were all outlined by Prof. Waksman. A notable feature of his address was the glimpse it gave of Ehrlich's method as a scientific worker and of his personal character.

Fhrlich, said Prof. Waksman, left little to chance and believed strongly in careful planning of research ; but he had the ability to use effectively unexpected observations. It happened, for example, that his kitchen-maid forgot to remove from the stove some stained preparations of tubercle bacilli, and this led
Ehrlich to realize the value of heating slides stained with certain dyes. It was, however, his systematic driving power and faith in finding the right answer that led, in Prof. Waksman's opinion, to Ehrlich's greatest discoveries. He represents, said Prof. Waksman, the "true scientist who does not sit idly by in deep contemplation waiting until a chance will come his way, but who searches for information in every branch of science that can only elucidate his particular problem".

Always an optimist, Ehrlich struggled throughout his life for the advance of human health. His temperament was hasty ; but he was also very kind. He always gave his assistants full credit for their work. Friendly and jovial to all around him, he neglected his own health, ate irregularly, worked and smoked continually and was indifferent to the comforts of life. Richard Willstätter, who went, in 1914, to Frankfurt to take part in the celebration of Ehrlich's sixtieth birthday, wrote that Ehrlich's "working cabinet" and laboratory were small and that the chairs and tables were covered with books, reprints, memoranda, flasks and tubes of every possible form, and cigar boxes in which were either imported cigars or tubes full of chemical preparations. Ehrlich knew and could find everything that was there, even if it took him a long time to look for it. The imp that was in him, which made him make joking remarks with an absolutely serious face and his habit of making fun of himself, confused-so said Miss Marquand, his secretary-people who were not used to that kind of thing. But the man, as we see him through these isolated comments, must indeed have been the man of whom Prof. Bulloch said : "Ehrlich was the most extraordinary man I ever met; with his wonderful inspiration, his enormous power of work, his astonishing knowledge, he was the greatest man in the medical world of his time. He was modest, sincere, noble-minded, and with the greatest kindness and consideration towards everybody".

At the present time, when, to use Prof. Waksman's words, "so much energy is being spent for machines of destruction", we do well to remember that Ehrlich, and the many others, alive and dead, who resemble him, are the true representatives of the scientific spirit. The world, which benefits from their work and nowadays demands these benefits as elementary human rights for which nothing, not even gratitude, need be paid, should be told much more about those who have given, and are giving, their busy lives to the single-minded service of their fellow men.

\section{G. LAPAGE}

\section{ASPECTS OF FOAM FORMATION}

$A^{T}$ $T$ the concluding 1951 meeting of the Society of Cosmetic Chemists of Great Britain, Dr. Raphael Matalon gave a lecture on "Some Aspects of Foam Formation". This was of particular interest because of Dr. Matalon's long experience in the study of molecular interactions at interfaces and his ingenious investigations into the foaming properties of detergent solutions, which were first undertaken at the University of Lyons. Following a period of fruitful collaboration with Dr. J. H. Schulman and others, in the Department of Colloid Science, University of Cambridge, he began his present work, on visco-elastic media and related phenomena, at King's College, Juondon, where he 
is currently directing research under the supervision of Sir Eric Rideal.

Dr. Matalon began by discussing, with frequent blackboard illustrations, the techniques that he uses for the study of foams, bubbles and laminx. He went on to deal with the chemical and structural properties connected with foam formation and foam stability, referring to the phenomena of molecular orientation, packing of molecules and structure in the adsorbed phase. There is evidence of liquid crystal formation and of a highly organized state in the bubble walls. He spoke next of elasticity and plasticity in foams and bubbles. Of essential importance for the stability of foams are those factors which control the rate of drainage of the interlamellar liquid. To cosmetic chemists, whose work on bath fluids and shampoos brings them into close touch with problems of foam formation, Dr. Matalon's remarks on interlamellar drainage and stability were of special interest, as were also, in certain other respects, his comments on the physicochemical properties related to anti-foaming.

Much of the subsequent discussion was concerned, in fact, with the problem of selecting or devising anti-foaming agents for use with specific systems (for example, sodium oleate in saponin solutions), in cases where foaming or excessive foaming is regarded as undesirable. Defoamers, it was emphasized, are simply disorganizers of surface structure. Dr. R. H. Marriott referred to the defoaming action for sodium alkyl sulphate solutions of traces of sodium stearate; while, speaking more generally, Mr. Jack Pickthall spoke of the many cosmetic applications of a 15 per cent solution of wool wax fatty acids in mineral oil as a defoaming agent. Dr. Matalon concluded the discussion by affirming that specific defoamers can readily be devised, and that many suggested by him have already been proved highly successful when used in relatively minute proportions.

A further talk was given by Mr. A. L. Waddams, of Shell Chemicals, Ltd., who dealt principally and at some length with the practical relationship of foaming and foam stability tests to the actual detergent effect of surface-active solutions on soiled fabrics.

F. V. WELLS

\section{FUNDAMENTAL ASPECTS OF LUBRICATION}

$\mathrm{T}$ $\mathrm{HE}$ subject of lubrication is one of interest to the physicist, chemist and engineer, as it involves a knowledge of the physical, chemical and mechanical properties of liquid films and metal surfaces. It is not surprising, therefore, that the topics discussed in the thirteen papers presented at the conference on "The Fundamental Aspects of Lubrication" held by the Section of Physics and Chemistry of the New York Academy of Sciences during March 3-4, 1950, and the text of which has recently been published (Ann. New York Acad. Sci., 53, Art. 4, pp. 753-994, June 27,$1951 ; 4$ dollars) cover a wide field. The conference was under the chairmanship of Dr. O. A. Beeck, of the Shell Development Co., and an outstanding figure in lubrication research, whose untimely death from a heart attack on July 5, 1950, is greatly to be deplored. It is fitting that the monograph covering the conference proceedings is dedieated to his memory. In addition to a photograph of Dr. Beeck as frontispiece, Prof. H. Mark, of the Brooklyn Polytechnic Institute, contributes a brief obituary in which he refers to Dr. Beeck as an ingenious research worker, a stimulating lecturer and a creative writer.

In the first three papers, recent developments in full-fluid lubrication, fundamental mechanical aspects of thin-film lubrication and the influence of surface films on the function, adhesion and surface damage of solids are dealt with. J. T. Burwell, of the Massachusetts Institute of Technology, explains that fullfluid lubrication generally means the condition of operation of a bearing where the surfaces are completely separated by a bulk film of lubricant so that no solid contact takes place. Both hydrodynamic lubrication, where the surfaces have either tangential or normal relative motion, and hydrostatic lubrication, where the surfaces are relatively at rest, are included. Side leakage and film rupture in journal bearings, bearings under dynamic loading and the effects of laminar and turbulent lubricant flow are also considered; and as an illustration of the spectacular application of hydrostatic lubrication, Dr. Burwell refers to the Mt. Palomar 200-in. telescope, the total weight of which, one million pounds, is supported at three points by hydrostatic pads and for which a torque of only $50 \mathrm{ft}$. $\mathrm{lb}$. is required to enable the telescope to follow the stars.

Thin-film lubrication, discussed by $\mathbf{H}$. Blok, of the Koninklijke/Shell Laboratory, Delft, lies between the two extremes of hydrodynamic lubrication and boundary lubrication. In the latter the load is fully borne by direct contact between the rubbing surfaces, and some aspects of this case, where the contact/load ratio is very high, are discussed in the third paper, by an acknowledged authority on this subject, Dr. F. P. Bowden, of the Department of Physical Chemistry, University of Cambridge. He reviews recent experimental investigations undertaken in his laboratory and discusses the bearing of the results obtained on the theory of friction.

Other papers in the monograph deal with X-ray and electron-diffraction studies of lubricating surfaces; surface chemical phenomena; physically and chemically adsorbed films in the lubrication of graphite sliding contacts; viscosity and molecular structure, in which the basic relations which exist between molecular structure, intermolecular forces and viscosity are developed in order to act as a guide for the preparation of synthetic lubricants; the measurement of the incremental friction coefficient of certain hydrocarbon compounds ; the influence of roughness and oxidation on wear of lubricated metal surfaces; and the fine structure of lubricating greases. Two papers on the complex process of metal cutting are of considerable interest. The process involves two main problems-the plastic deformation and failure of metals, and the friction and wear of sliding surfaces; thus it provides a convenient means for the study of the frictional properties of metals in sliding contact.

In the concluding paper in the monograph the fundamentals of lubrication studied by the aid of large metal crystals are described by $\mathbf{A}$. T. Gwathmey. The results of experiments to determine the action of gases and liquids on the single crystals, in the form of spheres and polished electrolytically, and the effect of static friction and wear between two base crystals of copper are discussed. The importance of the crystal face in lubrication chemistry and in friction and wear processes is clearly demonstrated. 